\title{
Perinatal arrhythmias
}

\author{
Nicole Sekarski • Erik Jan Meijboom • \\ Stefano Di Bernardo • Tatiana Boulos Ksontini • \\ Yvan Mivelaz
}

Received: 13 December 2013 /Revised: 5 March 2014 / Accepted: 3 April 2014 /Published online: 17 April 2014

(C) Springer-Verlag Berlin Heidelberg 2014

\begin{abstract}
Cardiac arrhythmias are very frequent in fetuses and newborns. The prognosis depends on the nature of the arrhythmias but is most often either spontaneously benign or following short-term medication administration. A correct diagnosis is essential for both management and prognosis. It is based on echocardiography during the fetal period and mainly on history, physical exam, and electrocardiogram after birth, but other modalities are available to record transient arrhythmic events. Irregular rhythms are mostly benign and rarely require therapy. In most fetuses and infants, tachyarrhythmias resolve spontaneously or require short-term administration of antiarrhythmics. Approximately one third of these may recur later on, especially during adolescence. Persistent bradyarrhythmias might require pacemaker implantation when associated with failure to thrive or with risk of sudden death. Conclusion: Arrhythmias in fetuses and infants are very common and mostly benign. History, physical exam, and recording of the arrhythmia are essential to make a correct diagnosis and establish an appropriate management for the rare potentially harmful arrhythmias.
\end{abstract}

Keywords Arrhythmia $\cdot$ Fetus $\cdot$ Newborn $\cdot$ Infant $\cdot$ Extrasystole $\cdot$ Tachycardia

Communicated by Patrick Van Reempts

N. Sekarski • E. J. Meijboom • S. Di Bernardo • T. B. Ksontini • Y. Mivelaz $(\bowtie)$

Cardiology Division, Pediatrics Department, Centre Hospitalier Universitaire Vaudois and University of Lausanne, Route du Bugnon 46, Bureau BH11/632, 1011 Lausanne, Switzerland e-mail: Yvan.Mivelaz@chuv.ch

E. J. Meijboom

Current affiliation: Pediatric Cardiology, University of Twente, Enschede, Netherlands

\author{
Abbreviations \\ ABC Airway, breathing, circulation \\ AET Atrial ectopic tachycardia \\ APB Atrial premature beat \\ AV Atrioventricular \\ AVB Atrioventricular block \\ AVNRT Atrioventricular nodal reentrant tachycardia \\ AVRT Atrioventricular reentrant tachycardia \\ CHD Congenital heart disease \\ ECG Electrocardiogram \\ JPB Junctional premature beat \\ JET Junctional ectopic tachycardia \\ PALS Pediatric advanced life support \\ PJRT Permanent junctional reciprocating tachycardia \\ SVT Supraventricular tachycardia \\ VPB Ventricular premature beat \\ VT Ventricular tachycardia \\ WPW Wolff-Parkinson-White syndrome
}

\section{Introduction}

Arrhythmias occur as soon as the heart starts to beat and end on a final irreversible arrhythmia when we die. The same definition, classification, and therapies apply from fetuses to adulthood. They are noted in about $2 \%$ of the pregnancies and account for 10 to $20 \%$ of the referrals to fetal cardiology units $[13,20]$. The prevalence of arrhythmias in normal infants (child up to the age of 12 months) is difficult to estimate, ranging from 0.75 to $14 \%$, and is higher in children with congenital heart disease (CHD) [7,32]. Arrhythmia is also a frequent cause of pediatric cardiology referrals in order to rule out potentially life-threatening conditions. We review here the classification, the diagnosis, the management, and the 
outcome of arrhythmias in fetuses and newborns (child under 28 days of age).

\section{Conduction system development}

The first heartbeat occurs by 3 weeks post-conception when the heart is only a primitive tubular structure. Major morphological remodeling occurs simultaneously with the development of the cardiac conduction system, which results by 7 weeks of gestation in a four-chambered heart with synchronous contraction of the atrial and ventricular chambers at a rate of approximately $110 \mathrm{bpm}$. Progressively, the sinus node acts as the primary pacemaker and the heart rate reaches $170 \mathrm{bpm}$ by 9 to 10 weeks. Later on in gestation, heart rate slowly decreases. Between 20 and 40 weeks of gestation, the heart rate is regular, with a range from 110 to $180 \mathrm{bpm}$ and a maximal beat-to-beat variation of $15 \mathrm{bpm}[20,44]$. After birth, heart rate slowly decreases and normal data for all electrocardiogram (ECG) time intervals including heart rate and wave axis have been published (Table 1) [9].

\section{Rhythm analysis}

During fetal life, a real-time fetal ECG is not obtainable due to the parasitic electrical field generated by the maternal heart and abdominal muscles. Magnetocardiography allows recording of the fetal heart magnetic field instead of the traditional electric field recorded by ECG. It is actually the best modality to analyze the fetal heart rhythm $[36,48]$. However, this technology is restricted to select centers due to its high cost. Therefore, the analysis of arrhythmias is routinely based on the ultrasound assessment of the temporal relationship of atrial and ventricular contractions. Different ultrasound modalities (M-mode, Doppler, or tissue Doppler) allow the classification of the arrhythmias (Fig. 1) $[6,13,40]$. In some cases, it may even be superior to ECG for arrhythmia assessment, as atrial contraction can be identified even when the ventricle is contracting or during the repolarization phase [37]. A systematic analysis of the ultrasound tracing is mandatory. Stepwise interpretation of the fetal heart rhythm is based on the determination of rhythm origin, regularity, and relationship between atrial and ventricular events and rate. Normal fetal rhythm includes an atrial origin, a regular atrial and ventricular contraction in a 1:1 fashion, and a normocardic heart rate.

In newborn and infants, analysis of the rhythm relies essentially on the surface ECG. ECG interpretation should follow a similar initial approach with assessment of rhythm origin, regularity, relationship, and rate (mnemonic: $4 \mathrm{R}$ ). In addition, a careful analysis of each wave (P, QRS, and T) and segment (PR, QT) in each lead will add information on the type of arrhythmia. For both diagnosis and management, it is

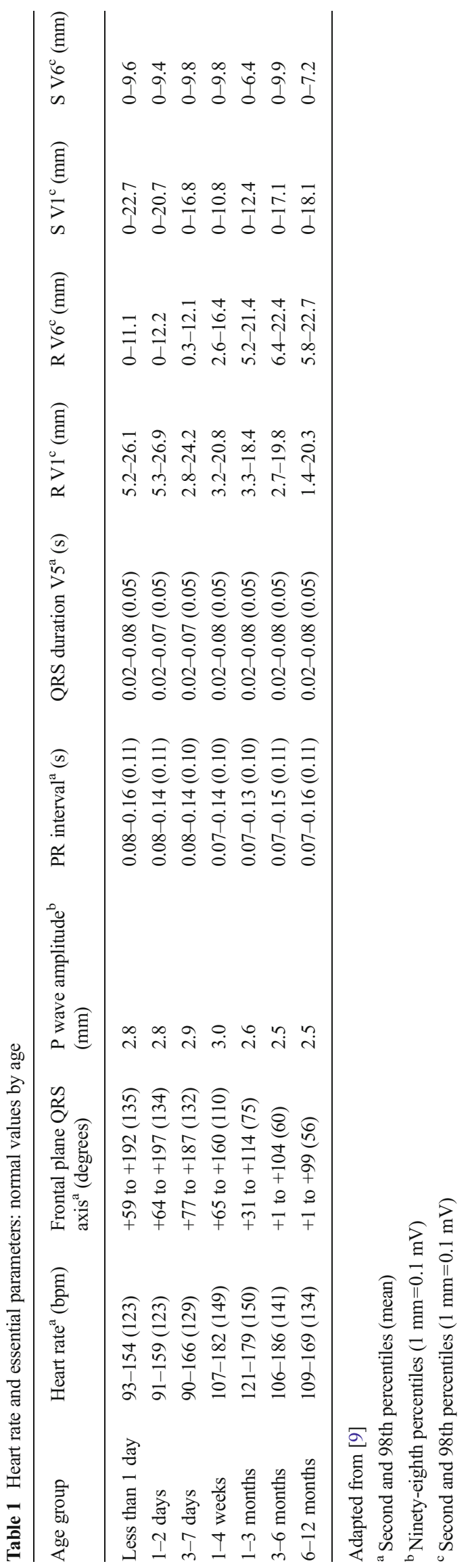



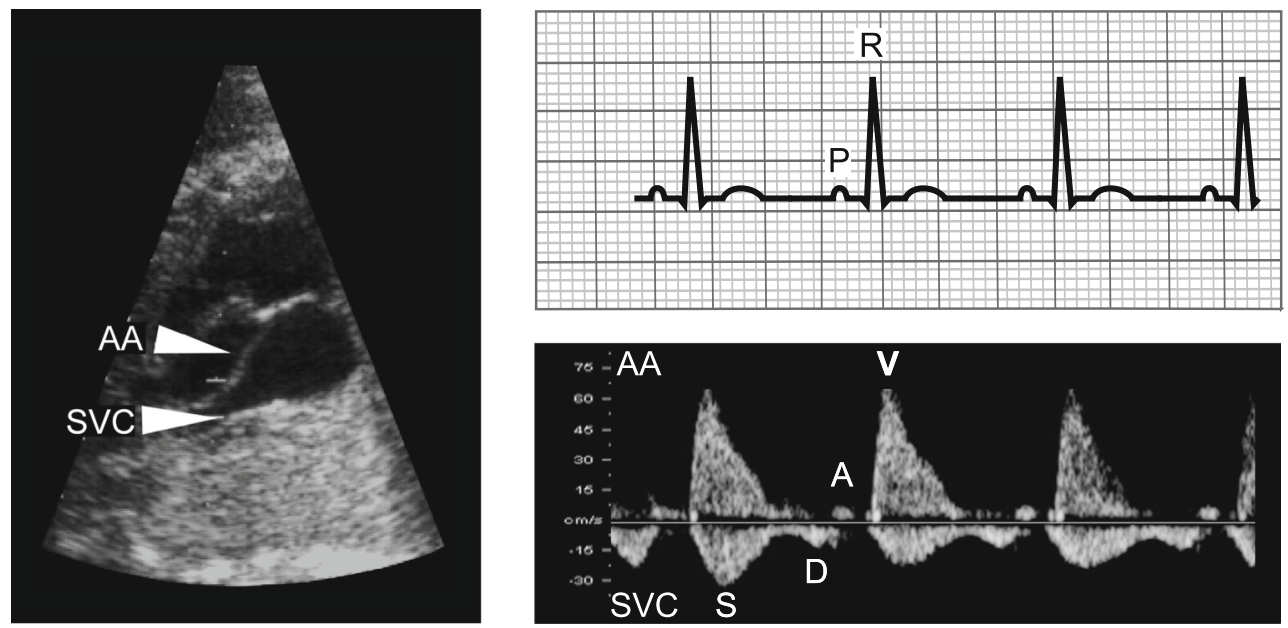

Fig. 1 Left, Two-dimensional ultrasound image showing the anatomical proximity of the SVC and the AA. The sample gate can easily be placed so as to record both proximal parts of SVC and AA. Right, Fetal virtual ECG (upper panel) and SVC/AA Doppler recording (lower panel, Doppler tracing turned upside down in order to illustrate analogies with the ECG tracing) in normal sinus rhythm. Ventricular ejection (V) in the AA

essential to distinguish a wide or a narrow QRS. Preexcitation (delta wave) or prolonged QT interval should also be ruled out.

\section{Irregular rhythm-premature beats}

\section{Definition}

A premature beat, also called premature contraction, ectopic beat, or extrasystole can originate from the atria, the atrioventricular junction, or the ventricle, and bypasses the sinus node. It is by far the most common type of arrhythmia encountered during the perinatal period, and their incidence in healthy patients varies greatly depending of the duration of the heart rhythm assessment from $1 \%$, when based on a single surface ECG, up to more than $50 \%$ when based on a 24 -h heart rate monitoring [17,46].

Atrial premature beats (APB)

\section{Incidence}

APB account for about $90 \%$ of irregular rhythm in fetuses and infants [17,22].

\section{Diagnosis}

On a Doppler or an ECG recording, APB is defined as a premature beat originating from the atria, which might be either conducted (generating a ventricular contraction) or blocked (not conducted through the atrioventricular (AV) appears above baseline. Venous flow is typical with systolic (S) and diastolic (E) waves below baseline and the retrograde flow wave (A) due to the atrial contraction above. $A$ late diastolic wave due to atrial contraction, $A A$ ascending aorta, $A V$ atrioventricular interval, $D$ early diastolic wave, $E C G$ electrocardiogram, $P$ P wave, $R$ R wave, $S$ systolic wave, $S V C$ superior vena cava, $V$ ventricular ejection

node) (Fig. 2). Prenatally, the demonstration of the atrial origin of the extra beat is based on the analysis of the timing of the atrial contraction and the resetting phenomenon of the sinus node with APB resulting in less than full compensatory pause.

Postnatally, on ECG, $\mathrm{P}$ waves resulting from an APB (called $\mathrm{P}^{\prime}$ ) usually have a different morphology than the sinus $\mathrm{P}$ wave. The morphology of the QRS is also helpful since most of the time a narrow QRS with similar morphology than sinus QRS is noted following an APB. A $\mathrm{P}^{\prime}$ wave is sometimes visible in the preceding $\mathrm{T}$ wave (Fig. 2).

\section{Management}

As arrhythmias can be associated with cardiac malformations [50], it is important to ensure that a routine mid-trimester fetal screening scan for cardiac anomalies is performed. If the APBs are very frequent (more than 5 per minute), persisting for more than 3 weeks or associated with signs of cardiac failure or extracardiac anomalies, it is recommended to perform a detailed fetal cardiac ultrasound [13]. A weekly monitoring of the fetal heart rate is recommended as long as the arrhythmia persists to detect the onset of supraventricular tachycardia (SVT). Indeed, the susceptibility to cardiac failure in fetuses with SVT and the inability to observe clinical manifestations justify this strategy. After birth, if the medical history and the physical exam are free of any cardiac sign or symptom and the ECG is normal for age except for the premature beat, monitoring or further investigation are not recommended. Exceptions are frequent APB and APB arising from multiple foci seen as $\mathrm{P}$ waves with different morphologies on surface ECG [52]. 
Fig. 2 Upper panel, ECG showing one nonconducted premature atrial beat (black arrowheads) occurring during $\mathrm{T}$ wave after every conducted atrial beat called bigeminy. This results in a ventricular bradycardia with a ventricular rate of $70 \mathrm{bpm}$. Lower panel, SVC/AA Doppler recording showing the same phenomenon than on the upper panel with an APB (white arrowheads) occurring at the end of the ventricular contraction which did not result in a ventricular contraction. $A$ atrial contraction, $A A$ ascending aorta, $P \mathrm{P}$ wave, $R \mathrm{R}$ wave, $S V C$ superior vena cava, $V$ ventricular contraction
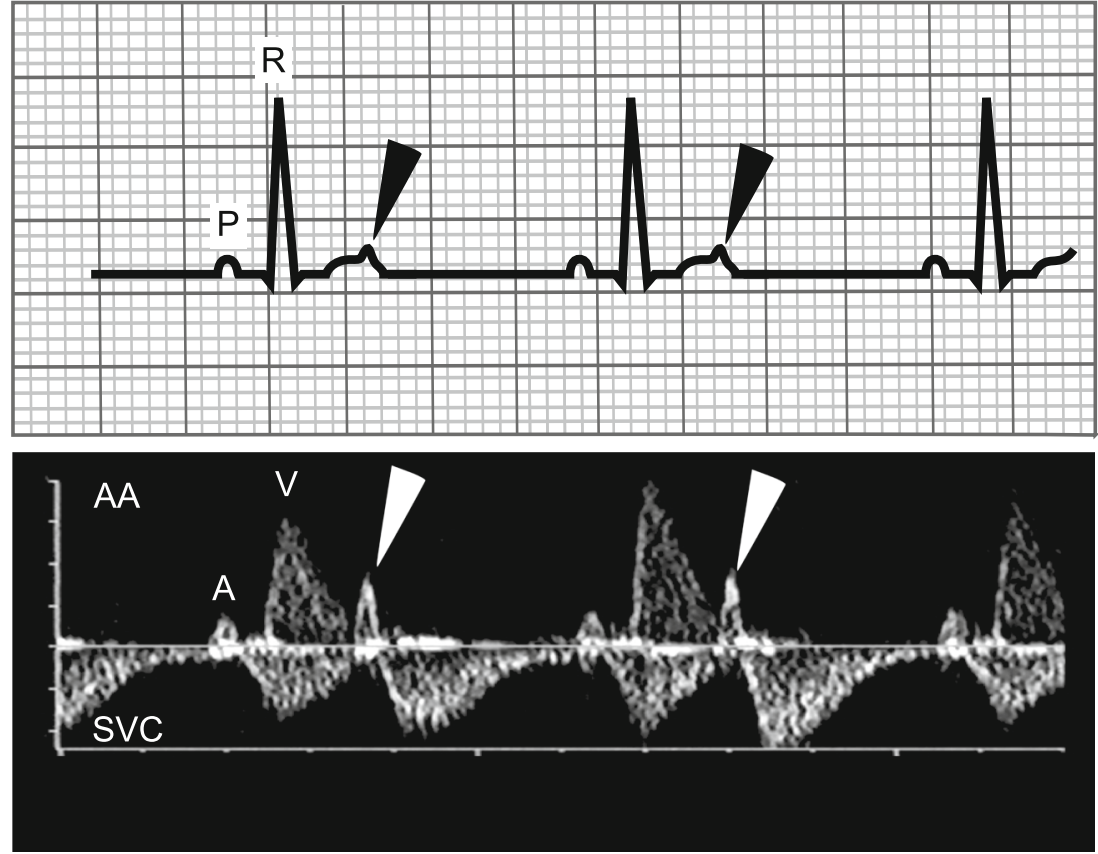

\section{Outcome}

APBs are mostly benign and remain self-limited with a spontaneous resolution after the diagnosis of the arrhythmia before birth in $95 \%$ of fetuses and by 1 year of age in $95 \%$ of children [46]. No association with sudden infant death or any other serious clinical problems has ever been described. The immaturity of conduction pathways in fetuses is characterized by an increased number of accessory pathways connecting the atrial and ventricular myocardium and a high rate of APB [31]. Both phenomena are responsible for the peak incidence of SVT by an atrioventricular (AV) reentrant mechanism during fetal life, which occurs in approximately 1 to $3 \%$ of fetuses with APB [13,20]. After birth, with the maturation of the conduction pathways, both APBs and SVTs tend to be self-limited [45]. Occasionally, APB can be associated with cardiac malformations, such as atrial septal defect. In sick babies, other causes should be ruled out: mechanical problems (central venous line, atrial distension), metabolic anomalies (hypo- or hyperkaliemia, hypercalcemia, hypoglycemia, hypoxia), pharmacological causes (antiarrhythmics, inotropes), or previous cardiac surgery (atrial scar).

Ventricular premature beats (VPB)

\section{Incidence}

VPB in fetuses are rare and represent less than $2 \%$ of fetal premature beats. After birth, the incidence of VPB in infants is highest in the neonatal period and decreases rapidly thereafter (incidence of VPBs on $24 \mathrm{~h}$ ambulatory ECG decreasing from
18 to $6 \%$, respectively) [17]. Therefore, some authors consider neonatal isolated monomorphic VPB a variation of normal rhythm [53].

\section{Diagnosis}

A VPB is diagnosed when, on a Doppler or an ECG recording, a premature ventricular beat is observed without any alteration of the atrial rate, giving rise to a full compensatory pause after the extra beat (Fig. 3). On ECG, a premature wide QRS, usually with a different morphology than the preceding QRS, makes the diagnosis easier postnatally.

\section{Management}

Prenatally, a careful exam of the cardiac anatomy and function should be performed to detect malformation or signs of cardiac failure. Weekly monitoring of the fetal heart rate is recommended as long as the arrhythmia persists [13,20]. After birth, besides a cardiovascular-oriented medical history and physical exam, an ECG should be obtained. But there is no agreement on the management of VPB diagnosed postnatally. Proposed managements are as follows: no further investigation, repetition of ECG at 1- or 2-month intervals, performance of a 24-h ambulatory electrocardiography monitoring to look for higher grade ectopic activity (nonisolated or polymorphic premature beats), and referral to a pediatric cardiologist for a cardiovascular work-up [27,52]. We believe that if medical history (personal and familial) and physical exam are free of any cardiovascular symptoms or signs, and if a pediatric cardiologist has ruled out abnormalities suggesting an 

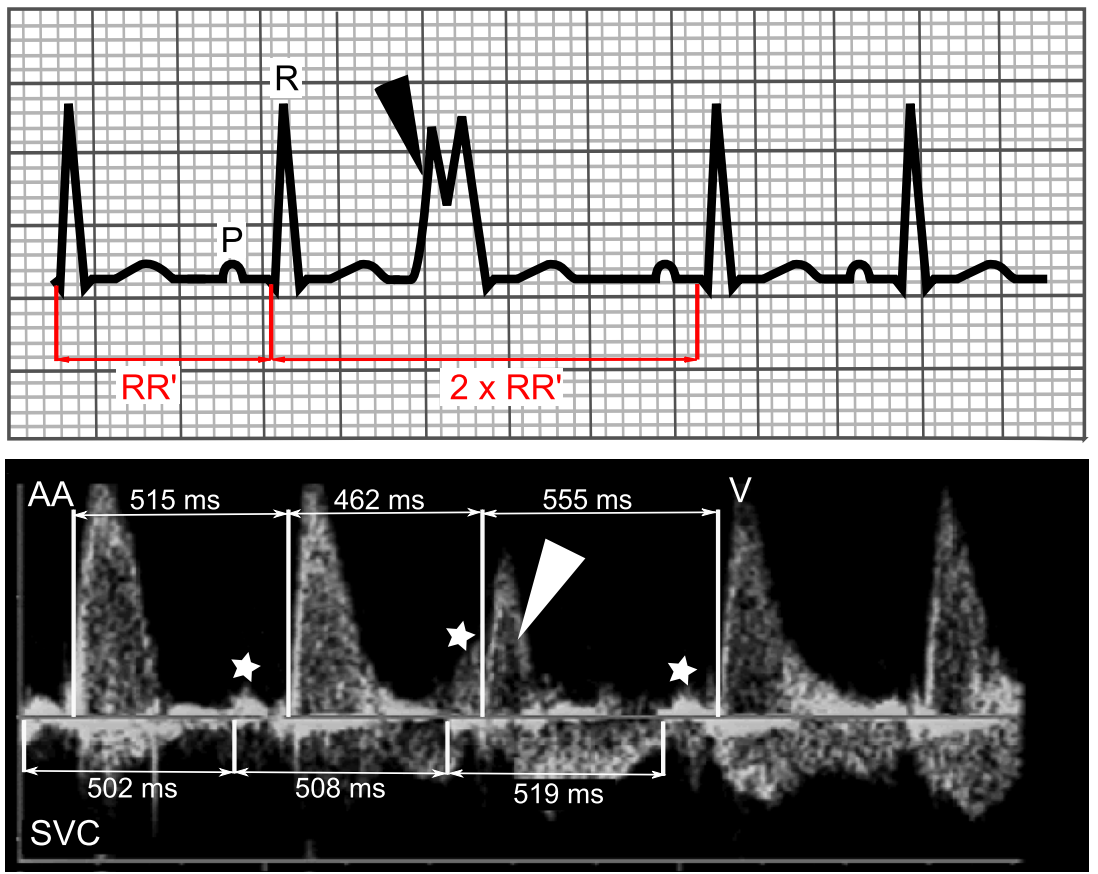

Fig. 3 Upper panel, surface ECG: VPB is diagnosed when a wide premature QRS complex (black arrowhead) is observed on surface ECG. Since in rare instances a wide QRS could result from an APB, another feature of VPB is that the interval between the preceding and the following sinus beat is equal to twice the time between two regular sinus beats. This phenomenon called "full compensatory pause" is due, in contrast to APB, to the absence of resetting of the sinus node in VPB. Lower panel, SVC/AA Doppler recording: prenatally, since ECG is not

underlying myocardial disease on ECG, it is reasonable to repeat the ECG beyond the neonatal period and perform a cardiovascular work-up for those who show persistent irregular rhythm at auscultation or ECG.

\section{Outcome}

The prognosis of VPB is also benign in most cases. However, it may be a manifestation of a serious underlying myocardial disease such as cardiac malformations, tumors, or cardiomyopathies. In most instances, those could be ruled out by medical history, physical examination, and ECG.

Junctional premature beats (JPB)

JPB describe premature beats originating from the AV node area. They are rarely described in fetuses and infants. The prenatal diagnosis is challenging because differentiation from VPB might be impossible. On ECG, an early narrow QRS not preceded by any $\mathrm{P}$ wave is diagnostic. Prognosis and management are similar to those with APBs [52]. obtainable, this phenomenon is essential to the diagnosis. $\mathrm{RR}^{\prime}$ interval before the VPB (white arrowhead) is $515 \mathrm{~ms}$. The interval from the preceding and the following regular ventricular beat equals $1,017 \mathrm{~ms}$, approximately twice the $\mathrm{RR}^{\prime}$ interval $(=1,030 \mathrm{~ms})$. Another feature is the identification of regular atrial contractions (white stars), independent of the premature ventricular contraction. $A A$ ascending aorta, $P \mathrm{P}$ wave, $R \mathrm{R}$ wave, $S V C$ superior vena cava, $V$ ventricular contraction

\section{Tachycardia}

\section{Definition}

Tachycardia is defined as an area of the heart that depolarizes faster than the normal range for age for at least three consecutive beats, above $180 \mathrm{bpm}$ in the fetus between 20 and 40 weeks of gestation and above $200 \mathrm{bpm}$ in infants. As for extrasystoles, supraventricular origin is by far the most frequent cause, with AV nodal and ventricular origins being infrequent in healthy fetuses and infants. In fetuses, $70 \%$ of the tachyarrhythmias are paroxysmal AV reentry tachycardia, $24 \%$ primary atrial tachycardias (mostly atrial flutter), and $6 \%$ sinus tachycardia [22]. During infancy, $80 \%$ of nonsinus tachycardia are AV reentry tachycardia, $15 \%$ are primary atrial tachycardia (mostly atrial flutter), and $5 \%$ are AV nodal reentry SVT [32]. The electrophysiological mechanism of all SVT is described in Table 2. During the perinatal period and infancy, a reentrant circuit is by far the most common mechanism. The reentrant circuit is either involving both the atria and ventricles, with the abnormal pathway crossing the AV groove in AV reentry tachycardia and within or just next to the AV node in AV nodal reentry tachycardia, or limited to the atria in atrial flutter $(\mathrm{AF})$. 


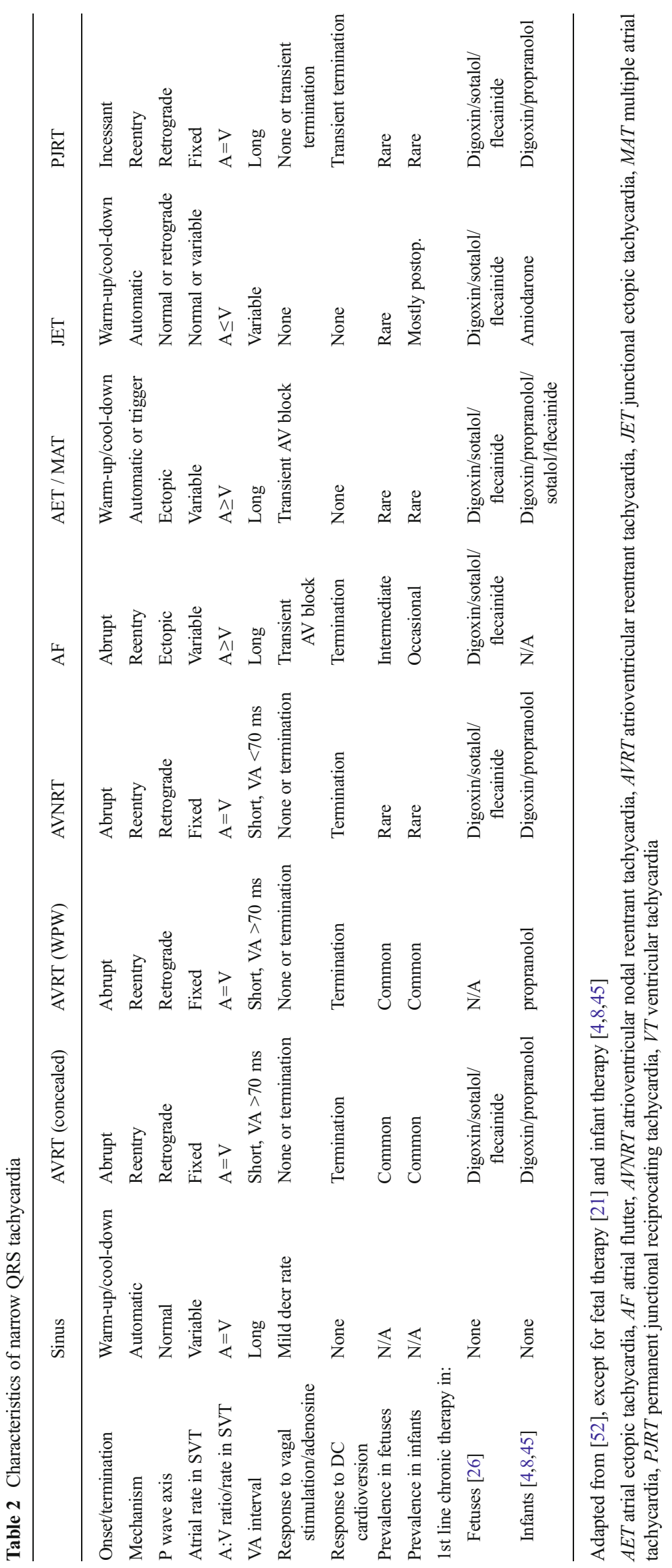


For the purpose of clarity, we will use a common terminology based on the depolarized cardiac chamber to characterize SVT on Doppler recordings and ECG. This means that instead of the traditional P, QRS waves, and PR interval, we will use the terms A (corresponding to atrial activity or event), $\mathrm{V}$ (corresponding to ventricular activity or event), and AV interval [52]. This allows to differentiate tachycardias based on the ratio of atrial versus ventricular events: $=1,>1$, or $<1$ and on the time interval between those events: VA interval $<\mathrm{AV}$ interval, called short VA SVT; or VA interval $>$ AV interval, called long VA SVT (Table 2; Fig. 4).

\section{Supraventricular tachycardia}

\section{Incidence}

The incidence of fetal SVT is about 1:3.700 pregnancies [50]. It accounts for about 5 to $10 \%$ of all fetal arrhythmias, but for more than $50 \%$ of the clinically significant ones [22]. Postnatally, the incidence ranges between 1:250 and 1:1,000 children, with a peak incidence in the neonatal period [17].

\section{Diagnosis}

The determination of the type of SVT is based on the assessment of the AV relationship and other specific characteristics (see Table 2 and Figs 4 and 5). Based on that, sinus tachycardia and ventricular tachycardia should be ruled out since their management and prognosis differ considerably from nonsinus SVT. Provoking factors for nonsinus SVT in the fetus have to be looked for: co-existing CHD, hyperthyroidism, or maternal caffeine, alcohol, or nicotine consumption. These last causes are among the most frequent ones [49]. Sinus tachycardia is the most common cause of long VA tachycardia and the heart rate is usually lower than in other SVT with frequent gradual acceleration and slowing. Atrial contractions remain always clearly visible on Doppler recording prenatally, but on ECG, P waves might be transiently hidden in the $T$ waves and visible only when the heart rate decelerates. Maternal fever, drug use, or hyperthyroidism and hypoxemia are the most frequent cause in fetuses. During infancy, the same etiologies are encountered, but hypovolemia due to dehydration or shock is the leading cause [18].

\section{Management}

Acute therapy Classification of SVT is the main step to establish a prognosis and guide the therapy. Prenatally, due to the risk of a rapid progression to cardiac failure and the difficulty to convert SVT in a hydropic fetus, all in utero sustained SVT should be treated when delivery is not an option $[13,20]$. Antiarrhythmic drugs have been used for years now and adverse events for the mother and the fetus remain rare. However, any drug administration to a healthy individu$\mathrm{al}$, the mother, is a great concern and careful introduction and monitoring is mandatory. The choice of the drug will depend mostly on the state of the fetus (signs of heart failure, fetal hydrops) as well as on the type of SVT. Since no large prospective randomized controlled trial (RCT) has been undertaken, there is to date no agreement on the best antiarrhythmic. This should be solved in the near future with the multicentric international RCT planned by Jaeggi et al. Because of their efficacy and safety, digoxin, sotalol, and flecainide are the first-line agents most commonly used (Table 2). All allow monitoring of the dosage based on their maternal serum level (serum level of sotalol and

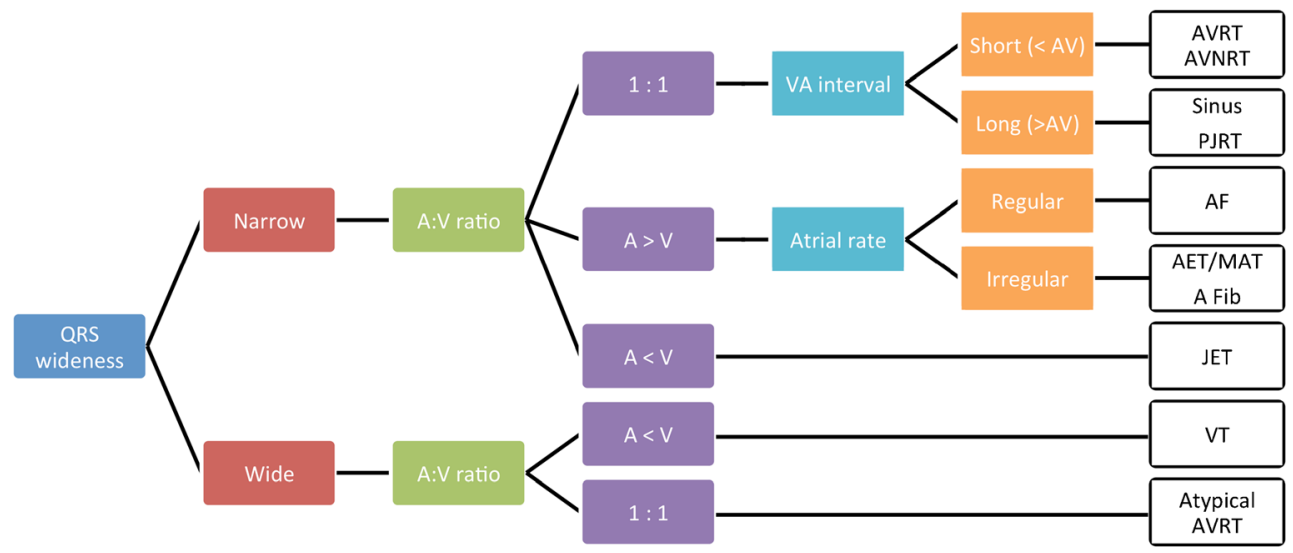

Fig. 4 SVT classification. After birth, QRS wideness allows to differentiate SVT from VT. The positive predictive value of a wide QRS for the diagnosis of VT is very high. Rare false positive cases are due to SVT with a bundle branch block or with antegrade conduction through an accessory pathway. Prenatally, without the availability of an ECG, tachycardia could still be differentiated based on the A:V ratio and the AV interval, except for differentiating JET from VT, both rare entities in healthy fetuses and newborn. $A E T$ atrial ectopic tachycardia, $A F$ atrial flutter, $A F i b$ atrial fibrillation, $A V R T$ atrioventricular reentrant tachycardia, AVNRT atrioventricular nodal reentrant tachycardia, JET junctional ectopic tachycardia, PJRT permanent junctional reciprocating tachycardia, Sinus sinus tachycardia, $V T$ ventricular tachycardia 

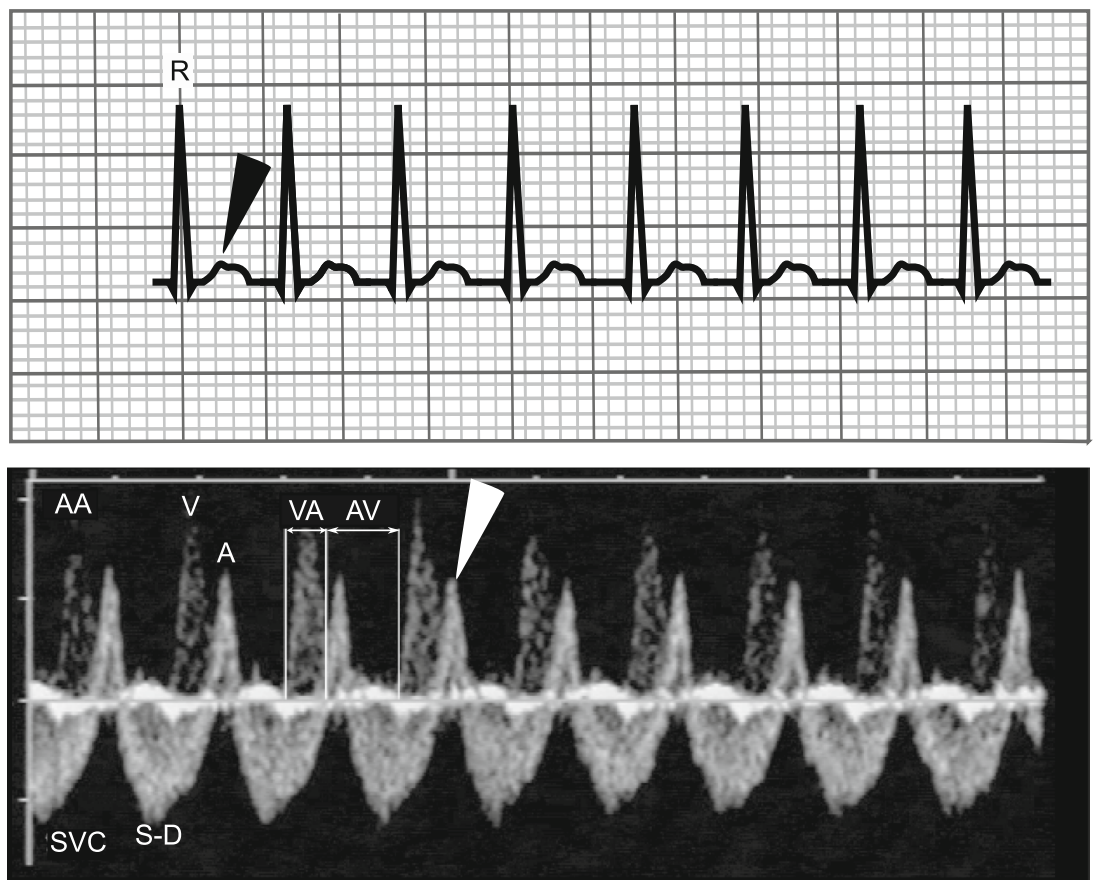

Fig. 5 Typical SVT with a short VA interval. On the surface ECG (upper panel), a narrow QRS tachycardia is observed. If we look carefully at the T wave, a little deflation (black arrowhead) can be observed sometimes corresponding to the retrograde depolarization of the atria. Prenatally (lower panel), a tachycardia is also noted on the Doppler recording based on the ventricular rate, but the atrial contractions are very clearly identifiable following shortly every ventricular depolarization. This phenomenon called canon A wave is due to the occurrence of the atrial contraction

flecainide might not be available in some centers) and the corrected QT measurement on maternal ECG. Combination of antiarrhythmics or second-line antiarrhythmics (propafenone, amiodarone) might be used in hydropic fetuses (Table 2). Indeed, in this situation, the conversion rate decreases to less than $25 \%$ with digoxin alone and usually a median of 2 medications are needed to convert to sinus rhythm [20]. Currently, the combinations of flecainide + digoxin and sotalol + digoxin are most commonly used. In severe cases, when all transplacental therapies have failed, direct administration of antiarrhythmic agents either through fetal intramuscular or transumbilical intravenous administration may be attempted.

The acute management of SVT in infants is based on pediatric advanced life support (PALS) guidelines from the American Heart Association [29]. Probable SVT with pulses and poor perfusion should be managed as follows: ABC status (i.e., airways, breathing, and circulation), oxygen delivery, cardiac monitoring, and IV access. If ECG recording confirms SVT and the patient is unstable, electrical synchronized direct current cardioversion 0.5 to $1 \mathrm{~J} / \mathrm{kg}$ has to be performed immediately. If the patient is hemodynamically stable, vagal maneuvers trial can be performed as long as adenosine administration is not delayed. Vagal maneuvers include diving before the opening of the tricuspid valve (the ventricle is still contracting and ejecting blood). The VA interval is much smaller than the AV interval, 83 and $171 \mathrm{~ms}$, respectively, for a heart rate of $240 \mathrm{bpm}$. $A$ late diastolic wave due to atrial contraction, $A A$ ascending aorta, $A V$ atrioventricular interval, $D$ early diastolic wave, $R$ R wave, $S$ systolic wave, $S V C$ superior vena cava, $V$ ventricular ejection, $V A$ ventriculoatrial interval

reflex and ice bag placement on the face of infants. A mix of crushed ice with water in a plastic bag or glove should be applied on the infant face for 10 to $15 \mathrm{~s}$, without compromising his breathing. In a stable patient, this might be repeated once. Carotid sinus massage and ocular pressure are not recommended due to prolonged asystole and potential retinal detachment, respectively. If not successful, adenosine should be given at a starting dose of $0.1 \mathrm{mg} / \mathrm{kg}$ (maximum $6 \mathrm{mg}$ ) intravenously by rapid bolus followed by saline flush administered as close to the patient as possible in the largest intravenous access. Conversion to sinus rhythm with the starting dose is frequently not obtained, especially in infants with less than $25 \%$ conversion with $0.1 \mathrm{mg} / \mathrm{kg}$ [11]. Therefore, the dose should progressively be increased up to $0.3 \mathrm{mg} / \mathrm{kg}$ (maximum $12 \mathrm{mg}$ ) [39]. Administration through umbilical artery catheter is the only contraindicated route due to the rapid metabolization of adenosine prior to its reaching the myocardium [30]. Adenosine acts by blocking the AV node and, therefore, only terminates arrhythmias involving the AV node such as AV reentrant tachycardia (AVRT) or AV nodal reentrant tachycardia (AVNRT). It will not terminate primary atrial tachycardias such as AF, but it will slow AV conduction and often unmask the underlying arrhythmia. It is therefore essential to record the ECG tracing during its administration. Adenosine is very effective for terminating SVT and also safe due to 
its short half life (less than $10 \mathrm{~s}$ ). Major side effects are infrequent, and they include severe bradycardia, asystole, atrial fibrillation, AV blocks, ventricular arrhythmias, and bronchospasm. Therefore, during its administration, a resuscitation cart has to be available at the bedside. In cases when adenosine is not recommended or unsuccessful, a pediatric cardiologist should be consulted for further management, either electrical cardioversion, overdrive pacing via esophageal leads or antiarrhythmic drugs administration. Acute heart failure is sometimes encountered in long-lasting tachycardia. In this situation, rapid restoration of sinus rhythm is crucial and vagal maneuvers and negative inotropes should be avoided. In this setting, digoxin is a valuable option for acute and long-term therapy.

$\mathrm{AF}$ is managed prior to birth by transplacental antiarrhythmic administration (Table 2, Fig. 4). Conversion to sinus rhythm is very effective with the administration of sotalol [34]. Postnatally, electrical cardioversion is the first-choice therapy either by atrial overdrive pacing or synchronized direct current cardioversion [3].

Long-term therapy Duration of therapy depends widely on the type of tachycardia and the difficulties encountered to convert it. Very little data exists on the postnatal management for fetuses diagnosed with SVT. Two strategies are mainly used either to discontinue all therapies at birth, or to continue antiarrhythmics prophylactically for 6 to 12 months. The first option is favored for short VA SVT easily converted with a single drug due to low recurrence rate and the second one for all other cases (long VA SVT, short VA SVT requiring more than one antiarrhythmic) [24]. Due to a very low recurrence rate after successful atrial flutter conversion, a chronic antiarrhythmic treatment is recommended neither in fetuses nor in infants $[24,34]$. However, it is important to teach parents the signs that might reflect a recurrent SVT: irritability, poor feeding, pallor, diaphoresis, and tachypnea. Since those signs might be subtle and also because of parental anxiety, some propose to teach parents to check the heart rate of their infant during sleep or feeding by either placing their hand on the child's chest or by using a stethoscope [28].

Postnatally, if a short VA SVT recurred or is newly diagnosed, a chronic therapy is usually initiated in infants to prevent further episodes. Drugs most used are digoxin and propranolol with similar efficiency [45]. However, since digoxin accelerates conduction through accessory pathways (AP), it is nowadays mostly avoided in children with WPW syndrome due to the risk of ventricular fibrillation in case of atrial fibrillation [16,32]. Other antiarrhythmics frequently used are sotalol, flecainide, propafenone, and amiodarone. Long-term therapy is usually discontinued after 6 to 12 months. In case of recurrency of SVT after discontinuation of medical therapy, the antiarrhythmic therapy is maintained for a longer period.
Other rare forms of SVT, like atrial ectopic tachycardia (AET), permanent junctional reciprocating tachycardia (PJRT), and junctional ectopic tachycardia (JET), are often more difficult to treat medically than short VA SVT. They respond poorly to acute management with adenosine administration, atrial overdrive pacing, or direct current cardioversion. They often require multiple therapies directed at controlling at least the rate of the tachycardia when converting to sinus rhythm is not achievable.

Until recently, radiofrequency ablation in children less than 5 years of age and weighing less than $15 \mathrm{~kg}$ was restricted to malignant or poorly tolerated arrhythmias [16]. With improving technologies and expertise, it is now a safe and effective alternative in infants [5]. Catheter ablation should be considered at any age for SVT associated with aborted sudden death, arrhythmiarelated syncopal episodes, ,ventricular dysfunction, refractoriness to multidrug therapy, or severe drug-related side effects $[3,5]$. This procedure should be performed only in experienced hands.

\section{Outcome}

Studies show that the recurrence rate of paroxysmal SVT is the lowest for prenatal cases and then inversely related to age at initial diagnosis [41]. Patients with initial SVT during infancy have a freedom from SVT at 1 year of age of $90 \%$, but approximately one third of them may have recurrences later in childhood. The reason for the low recurrence rate in prenatal cases lies probably with two phenomena: the progressive disappearance of the muscular accessory pathways connecting the atria and ventricles as the infant's heart grows and the low rate of premature beats as initiating events during infancy [19].

In the recent era, fetal SVT in hydropic fetuses is still associated with a significant mortality (as high as $17 \%$ ) and morbidity rate (neurological abnormalities reported in 10 to $20 \%$ of cases even after successful treatment) [23,38].

Ventricular tachycardia

\section{Incidence}

VT is very rare in fetuses and infants, accounting for less than $1 \%$ of all tachyarrhythmias and with an incidence of 0.3 episodes $/ 100,000$ infants [16,42]. An underlying structural heart disease (hypertrophic cardiomyopathy, long QT syndrome, right ventricular dysplasia, left ventricle noncompaction, congenital cardiac malformation) is present in approximately half of the pediatric cases. 


\section{Diagnosis}

Prenatally, the diagnosis is challenging, but should be suspected if AV dissociation is observed with more ventricular contractions than atrial ones on fetal echo. After birth, the diagnosis is made if, on ECG, there are more than three consecutive wide QRS beats of ventricular origin occurring at a faster rate than the underlying rhythm. A rare benign cause of broad QRS tachycardia in neonates is accelerated idioventricular rhythm, characterized by a broad QRS on ECG with a frequency rate slightly higher than the prevailing sinus rhythm in a perfectly asymptomatic patient. Spontaneous conversion to sinus rhythm might be observed when heart rate rises due to the infant agitation. Etiology is unknown, and it is always self-limited, benign, and never evolves toward a VT [14,42].

\section{Management}

Prenatal treatment includes beta-blockers, flecainide, sotalol, lidocaine, and amiodarone, but due to the very limited number of cases, success rate of treatment is not clearly established and a first-line agent remains to be established. Amiodarone and sotalol have proven to be successful, but there are concerns about their proarrhythmic side effects in case of associated LQTS. Therefore, some advocate the use of flecainide or propranolol, which decreases the QT interval and thus the risk of inducing torsades de pointes. Postnatally, management of sustained VT also follows the American Heart Association PALS guidelines: ABC status, oxygen delivery, cardiac monitoring, and IV access and ECG recording for rhythm assessment and electrical synchronized cardioversion 0.5 to $1 \mathrm{~J} / \mathrm{kg}$. Hemodynamically stable infants with VT might be managed by drug administration with lidocaine or amiodarone [10,33]. In the setting of prolonged QT and torsades de pointes (polymorphic ventricular tachycardia), intravenous magnesium sulfate is the treatment of choice. The long-term management still has to be established since, without the occurrence of lifethreatening events or death, VT resolution occurs with and without outpatient antiarrhythmic medication [33].

\section{Outcome}

The outcome of VT depends on its etiology. Congenital heart disease, cardiomyopathies (hypertrophic, arrhythmogenic right ventricular cardiomyopathy), long QT syndrome, and cardiac tumors are associated with a poor outcome. The mortality reported is $36 \%$ in this population [42]. Inversely, in half of fetuses and infants, VT is observed in apparently healthy individuals and is associated with a good prognosis $[10,33,42]$.

\section{Bradycardia}

\section{Definition}

Bradycardia is defined as an area of the heart that depolarizes slower than the normal range for age for at least three successive beats. A ventricular rate in fetuses and infants below $110 \mathrm{bpm}$ and below $100 \mathrm{bpm}$, respectively, is usually considered bradycardia $[9,13]$.

Incidence

Bradycardia represents less than $5 \%$ of arrhythmia referral in fetuses and infants [13].

\section{Etiology}

The most common cause is sinus bradycardia. Transient sinus bradycardia is often secondary to vagal stimulation, frequently occurring during fetal scan as a result of the pressure applied to the maternal abdomen and is also noted during deep sleep, vomiting, and defecation in infants [47]. Persistent sinus bradycardia is often related to cardiac failure and hydrops in fetuses, but might also be caused by either maternal hypothermia or long QT syndrome. In infants, sustained sinus bradycardia is mostly related to secondary sinus node dysfunction (Table 3). Nonsinus bradycardia consists of sinus node dysfunction either idiopathic or related to a CHD pre- or postrepair, blocked atrial bigeminy (Fig. 2), 2:1 second-degree atrioventricular block (AVB), or complete AVB. Atrioventricular blocks will be discussed separately in the next section.

\section{Diagnosis}

In utero atrial and ventricular contraction rate and relationship allows identification of the type of bradycardia, either sinus bradycardia, blocked atrial bigeminy, 2:1 AVB or complete AVB (Fig. 2). Postnatally, the diagnosis is easily made on surface ECG.

\section{Management}

For all bradycardias, management depends on etiology and severity, but a close follow-up is always necessary in order to detect the occurrence of cardiac failure.

\section{Outcome}

Usually, blocked atrial bigeminy is not associated with the development of cardiac failure and converts spontaneously to sinus rhythm. The outcome of sinus bradycardia depends highly on the underlying cause. 
Table 3 Causes of sinus bradycardia in fetuses or infants

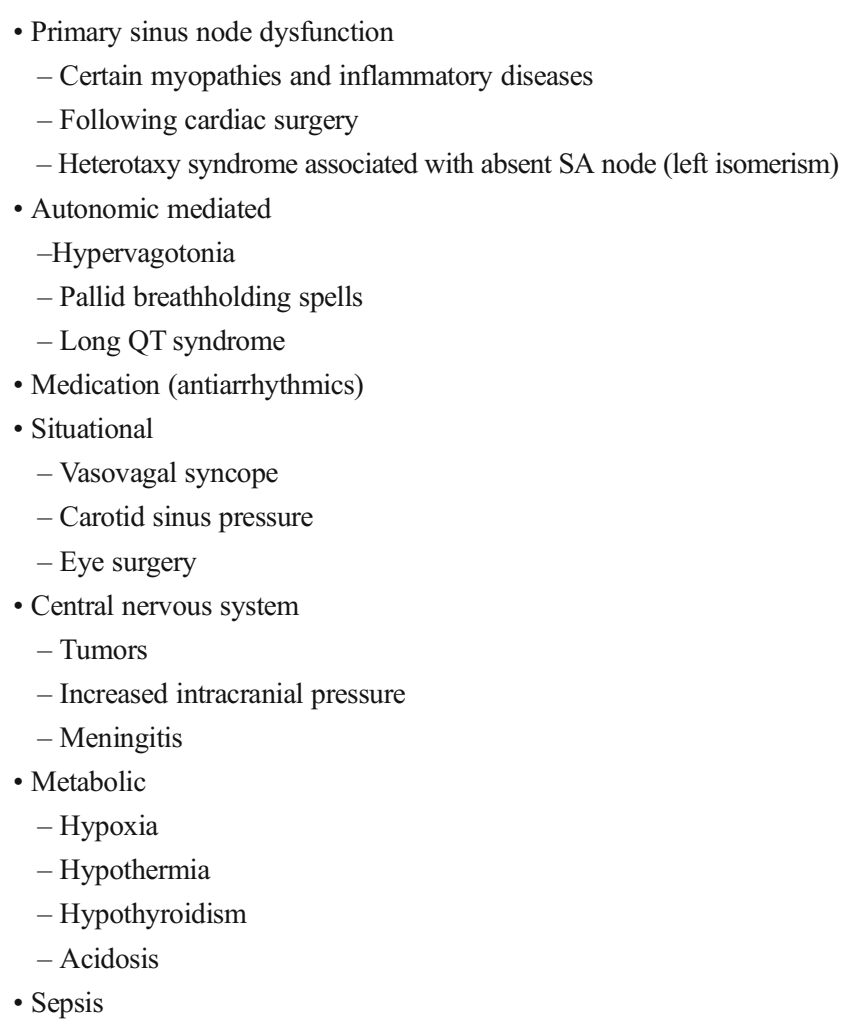

Adapted from [52]

\section{Atrioventricular conduction disorders}

\section{Complete AV block}

\section{Definition}

Complete AVB is the complete dissociation between atrial and ventricular contractions. Atrial and ventricular impulses are generated independently from each other. Complete AVB occurring prenatally are equally related to either CHD (left isomerism, atrioventricular septal defect, corrected transposition of the great arteries) or maternal autoantibodies (associated with autoimmune diseases such as systemic lupus erythematosus) $[35,47]$. Postnatally, the majority is acquired after surgery for CHD, but carditis (viral myocarditis and Lyme diseases carditis essentially) and idiopathic causes might be encountered [35,52].

\section{Diagnosis}

The diagnosis is easily made prenatally and postnatally by identifying atrial and ventricular events, on Doppler or ECG recording, occurring independently from each other at their own pace.

\section{Management}

When delivery is not an option, therapeutic choices are limited. It has been suggested that immune-related second-degree and complete AVB might benefit from transplacental steroid administration to prevent AV node and fetal myocardial damage, but this is still controversial $[21,26,35,43]$. Significant in utero morbidity and mortality has been reported related mainly to cardiac failure and hydrops, which occurs in 9 to $27 \%$ of the pregnancies with a higher rate in those fetuses with associated CHD $[25,35]$. Since with a heart rate below $55 \mathrm{bpm}$ the prognosis seems very poor, therapies aiming at increasing heart rate have been proposed. Transplacental salbutamol showed some effects in increasing the fetal ventricular rate and case reports describe the use of in utero pacing, but the clinical benefit of these therapies remains to be demonstrated $[2,21,26,43]$.

In contrary to prenatal management, postnatal management is more clearly established due to the possibility of pacemaker implantation. Advanced second- or third-degree AVB following surgery and persisting more than 7 days requires pacemaker implantation. Nonsurgical advanced second- or thirddegree AVB might be followed conservatively. Criteria for pacemaker implantation in those patients include the following: failure to thrive, cardiovascular symptoms related to the bradycardia, low cardiac output, and resting heart rate during infancy less than $55 \mathrm{bpm}$ [5]. Finally, any infant with symptomatic sinus bradycardia or chronotropic incompetence should be referred for pacemaker implantation [5].

\section{Outcome}

Complete AVB with co-existing CHD carries the worst prognosis with only 20-40\% survival beyond the neonatal period [35]. One-year survival for isolated perinatal complete AVB is between 80 and $90 \%$. Dilated cardiomyopathy is observed in approximately $25 \%$ of the survivors [43,51].

\section{Second-degree AV block}

\section{Definition-diagnosis-management-outcome}

In second-degree AV block, not every atrial beat is conducted to the ventricles. The diagnosis is made similarly for all AV blocks by identifying the atrial and ventricular beats and the blocked atrial beat, and measuring the AV delay. In Mobitz type I subtype (Wenckebach), there is a progressive increase in AV conduction delay ending in a nonconducted atrial beat. This condition shares the same etiologies and prognosis as first-degree AVB. It is also a physiologic condition, frequently observed when vagal tone is more prominent, especially during sleep. However, progression to higher degrees of $\mathrm{AV}$ block is possible, especially if it is related to maternal 
autoantibodies exposition. Those have to be searched for prenatally and appropriate treatment should be established. An underlying cardiac condition is also possible and has to be excluded during the prenatal ultrasound and after birth by history and physical examination. Patient should be referred to a pediatric cardiologist and a 24-h ambulatory heart rate monitoring is indicated to observe if the condition is only transient and related with periods of high vagal tone. If so, follow-up is not necessary. For the remaining cases, etiologies should be looked for and a yearly follow-up is suggested.

In Mobitz type II second-degree AV block, there is no progressive lengthening of the $\mathrm{AV}$ conduction, but rather a sudden interruption of $\mathrm{AV}$ conduction. This is a rare but more serious condition than type I, often related either to inflammation (maternal autoantibodies) or traumatic injury. There is a higher risk for progression to complete AVB, especially for advanced second-degree AV block (defined as the blocking of two or more consecutive $\mathrm{P}$ waves). Therefore, pacemaker implantation is indicated when such block occurs following surgery [5]. Its incidental finding in an asymptomatic patient can be managed conservatively, but deserves careful follow-up.

First-degree AV block

\section{Definition-diagnosis-management-outcome}

In first-degree AV block, every atrial beat is conducted to the ventricles with some delay. The diagnosis is based on the measurement of a prolonged AV or PR interval for age on Doppler recording or ECG. AV interval increases slightly through gestation and normal data varies depending on the technique used [1,12]. Normal PR interval is less than $160 \mathrm{~ms}$ the first day of life and decreases progressively down to $130 \mathrm{~ms}$ at 3 months of age [1,9]. Then its upper limit rises up to $150 \mathrm{~ms}$ and to $160 \mathrm{~ms}$ from 3 to 6 and 6 to 12 months, respectively (Table 1).

First-degree AV block is a benign condition and is well tolerated. It does not require any therapy, but it might reflect damage from maternal autoantibodies to the conduction pathway. This should be looked for prenatally and followed appropriately [15]. A careful medical history and physical exam has to be performed to rule out other underlying cardiac diseases.

This is mostly a benign condition, physiological in most instances, encountered in infants with high levels of vagal tone, especially during sleep, but it can also be associated with CHD, antiarrhythmic medication, hypothyroidism, myocardial inflammation, or surgical trauma [52].

\section{Conclusion}

Arrhythmias in fetuses and infants are very common. History, physical exam, and recording of the arrhythmia are the cornerstones of the management. Doppler echocardiography and ECG are the preferred methods for the prenatal and postnatal periods, respectively. Premature beats are almost always benign. The most common SVTs, AV re-entrant tachycardia and atrial flutter, carry an excellent prognosis. Acute and chronic antiarrhythmic medications are available to treat and prevent recurrent SVT until spontaneous resolution of the tachyarrhythmia occur, as is the case in up to $90 \%$ of the treated fetuses and infants. If therapy fails, which is more common for rarer forms of SVT, electrophysiological study and catheter ablation could be an option. Due to the increased risks of such procedures in infants, it has to be undertaken in experienced hands. Complete AVB is a serious condition with significant morbidity and mortality, especially when associated with CHD and may need pacemaker implantation.

Acknowledgments Some Doppler recording tracings were provided courtesy of Jean-Claude Fouron MD, Pediatric Cardiology, Sainte Justine Hospital, Montreal, Canada.

Conflict of interest The authors do not have any conflict of interest regarding this publication.

\section{References}

1. Andelfinger G, Fouron JC, Sonesson SE, Proulx F (2001) Reference values for time intervals between atrial and ventricular contractions of the fetal heart measured by two Doppler techniques. AJC 88:1433$1436-\mathrm{A} 8$

2. Assad RS, Zielinsky P, Kalil R et al (2003) New lead for in utero pacing for fetal congenital heart block. J Thorac Cardiovasc Surg 126:300-302. doi:10.1016/S0022-5223(03)00220-4

3. Bauersfeld U, Pfammatter JP, Jaeggi E (2001) Treatment of supraventricular tachycardias in the new millennium - drugs or radiofrequency catheter ablation? Eur J Pediatr 160:1-9

4. Bonney WJ, Shah MJ (2013) Incessant SVT in children: ectopic atrial tachycardia and permanent junctional reciprocating tachycardia. Progress in Pediatric Cardiology 1-8. doi: 10.1016/j.ppedcard. 2012.11.005

5. Brugada J, Blom N, Sarquella-Brugada G et al (2013) Pharmacological and non-pharmacological therapy for arrhythmias in the pediatric population: EHRA and AEPCArrhythmia Working Group joint consensus statement. Europace 15:1337-1382. doi:10.1093/europace/eut082

6. Carvalho JS, Prefumo F, Ciardelli V et al (2007) Evaluation of fetal arrhythmias from simultaneous pulsed wave Doppler in pulmonary artery and vein. Heart 93:1448-1453. doi:10.1136/hrt.2006.101659

7. Chiu S-N, Wang J-K, Wu M-H et al (2008) Cardiac conduction disturbance detected in a pediatric population. J Pediatr 152:85-89. doi:10.1016/j.jpeds.2007.05.044

8. Collins KK, Van Hare GF, Kertesz NJ et al (2009) Pediatric nonpostoperative junctional ectopic tachycardia. JAC 53:690-697. doi:10. 1016/j.jacc.2008.11.019

9. Davignon A, Rautaharju P, Boisselle E et al (1980) Normal ECG standards for infants and children. Pediatr Cardiol 1:123-152

10. Davis AM, Gow RM, McCrindle BW, Hamilton RM (1996) Clinical spectrum, therapeutic management, and follow-up of ventricular tachycardia in infants and young children. Am Heart J 131:186-191 
11. Dixon J, Foster K, Wyllie J, Wren C (2005) Guidelines and adenosine dosing in supraventricular tachycardia. Arch Dis Child 90:1190 1191. doi:10.1136/adc.2005.077636

12. Epstein AE, DiMarco JP, Ellenbogen KA et al (2008) ACC/AHA/ HRS 2008 guidelines for device-based therapy of cardiac rhythm abnormalities: a report of the American College of Cardiology/ American Heart Association Task Force on Practice Guidelines (Writing Committee to Revise the ACC/AHA/NASPE 2002 Guideline Update for Implantation of Cardiac Pacemakers and Antiarrhythmia Devices): developed in collaboration with the American Association for Thoracic Surgery and Society of Thoracic Surgeons. Circulation 117:e350-408. doi:10.1161/ CIRCUALTIONAHA.108.189742

13. Fouron J-C (2004) Fetal arrhythmias: the Saint-Justine hospital experience. Prenat Diagn 24:1068-1080. doi:10.1002/pd.1064

14. Freire G, Dubrow I (2008) Accelerated idioventricular rhythm in newborns: a worrisome but benign entity with or without congenital heart disease. Pediatr Cardiol 29:457-462. doi:10.1007/s00246-007-9024-z

15. Friedman DM, Kim MY, Copel JA et al (2008) Utility of cardiac monitoring in fetuses at risk for congenital heart block: the PR Interval and Dexamethasone Evaluation (PRIDE) prospective study. Circulation 117:485-493. doi:10.1161/CIRCULATIONAHA.107. 707661

16. Friedman RA, Walsh EP, Silka MJ, et al. (2002) NASPE Expert Consensus Conference: radiofrequency catheter ablation in children with and without congenital heart disease. Report of the Writing Committee. North American Society of Pacing and Electrophysiology. In: Pacing Clin Electrophysiol. pp 1000-1017

17. Garson AJ, Gillette PC, McNamara DG (1981) Supraventricular tachycardia in children: clinical features, response to treatment, and long-term follow-up in 217 patients. J Pediatr 98:875-882

18. Gimovsky ML, Nazir M, Hashemi E, Polcaro J (2004) Fetal/neonatal supraventricular tachycardia. J Perinatol 24:191-193. doi:10.1038/sj. jp. 7211036

19. Hahurij ND, Gittenberger-De Groot AC, Kolditz DP et al (2008) Accessory atrioventricular myocardial connections in the developing human heart: relevance for perinatal supraventricular tachycardias. Circulation 117:2850-2858. doi:10.1161/CIRCULATIONAHA.107. 756288

20. Hornberger LK, Sahn DJ (2007) Rhythm abnormalities of the fetus. Heart 93:1294-1300. doi:10.1136/hrt.2005.069369

21. Jaeggi ET (2004) Transplacental fetal treatment improves the outcome of prenatally diagnosed complete atrioventricular block without structural heart disease. Circulation 110:1542-1548. doi:10.1161/01. CIR.0000142046.58632.3A

22. Jaeggi E (2009) Electrophysiology for the perinatologist. In: Yagel S, Silverman NH, Gembruch U (eds) Fetal cardiology, 2nd edn. Informa Healthcare London, New York, pp 435-447

23. Jaeggi ET, Carvalho JS, De Groot E et al (2011) Comparison of transplacental treatment of fetal supraventricular tachyarrhythmias with digoxin, flecainide, and sotalol: results of a nonrandomized multicenter study. Circulation 124:1747-1754. doi:10.1161/ CIRCULATIONAHA.111.026120

24. Jaeggi E, Fouron JC, Drblik SP (1998) Fetal atrial flutter: diagnosis, clinical features, treatment, and outcome. J Pediatr 132:335-339

25. Jaeggi ET, Hornberger LK, Smallhorn JF, Fouron JC (2005) Prenatal diagnosis of complete atrioventricular block associated with structural heart disease: combined experience of two tertiary care centers and review of the literature. Ultrasound Obstet Gynecol 26:16-21. doi:10. 1002/uog. 1919

26. Jaeggi ET, Silverman ED, Laskin C et al (2011) Prolongation of the atrioventricular conduction in fetuses exposed to maternal anti-Ro/ SSA and anti-La/SSB antibodies did not predict progressive heart block. JAC 57:1487-1492. doi:10.1016/j.jacc.2010.12.014

27. Kaltman J, Shah M (2004) Evaluation of the child with an arrhythmia. Pediatr Clin N Am 51:1537-51- viii. doi:10.1016/j.pcl.2004.08.002
28. Kantoch MJ (2005) Supraventricular tachycardia in children. Indian J Pediatr 72:609-619

29. Kleinman ME, Chameides L, Schexnayder SM et al (2010) Part 14: pediatric advanced life support: 2010 American Heart Association Guidelines for Cardiopulmonary Resuscitation and Emergency Cardiovascular Care. Circulation 122:S876-908. doi:10.1161/ CIRCULATIONAHA.110.971101

30. Knick BJ, Saul PJ (2001) Immediate arrhythmia management. In: Zeigler VL, Gillette PC (eds) Practical management of pediatric cardiac arrhythmias. Futura, Armonk, NY, pp 161-230

31. Ko JK, Deal BJ, Strasburger JF, Benson DW (1992) Supraventricular tachycardia mechanisms and their age distribution in pediatric patients. AJC 69:1028-1032

32. Lee KW, Badhwar N, Scheinman MM (2008) Supraventricular tachycardia - part I. Curr Probl Cardiol 33:467-546. doi:10.1016/j. cpcardiol.2008.06.002

33. Levin MD, Stephens P, Tanel RE et al (2010) Ventricular tachycardia in infants with structurally normal heart: a benign disorder. Cardiol Young 20:641-647. doi:10.1017/S1047951110000867

34. Lisowski LA, Verheijen PM, Benatar AA et al (2000) Atrial flutter in the perinatal age group: diagnosis, management and outcome. JAC 35:771-777

35. Lopes LM, Tavares GMP, Damiano AP et al (2008) Perinatal outcome of fetal atrioventricular block: one-hundred-sixteen cases from a single institution. Circulation 118:1268-1275. doi:10.1161/ CIRCULATIONAHA.107.735118

36. Mivelaz Y (2014) Electrophysiology of the fetal heart assessed by magnetocardiography: a hidden area revealed but with some limitations. Heart Rhythm. doi:10.1016/j.hrthm.2013.12.040

37. Mivelaz Y, Sarquella-Brugada G, Fournier A, Fouron J-C (2009) The underestimated potential of Doppler ultrasound to assess fetal arrhythmia: first report of a prenatal, transient, atypical atrioventricular block. Heart Rhythm 6:1226-1228. doi:10.1016/j.hrthm.2009.03.049

38. Oudijk MA, Gooskens RHJM, Stoutenbeek P et al (2004) Neurological outcome of children who were treated for fetal tachycardia complicated by hydrops. Ultrasound Obstet Gynecol 24:154 158. doi:10.1002/uog. 1106

39. RCPCH (2003) Medicines for children, 2nd edn. RCPCH Publications Limited, London

40. Rein AJJT (2002) Use of tissue velocity imaging in the diagnosis of fetal cardiac arrhythmias. Circulation 106:1827-1833. doi:10.1161/ 01.CIR.0000031571.92807.CC

41. Riggs TW, Byrd JA, Weinhouse E (1999) Recurrence risk of supraventricular tachycardia in pediatric patients. Cardiology 91:25-30

42. Roggen A, Pavlovic M, Pfammatter J-P (2008) Frequency of spontaneous ventricular tachycardia in a pediatric population. AJC 101: 852-854. doi:10.1016/j.amjcard.2007.10.047

43. Rosenthal E, Gordon PA, Simpson JM, Sharland GK (2005) Letter regarding article by Jaeggi et al, "Transplacental fetal treatment improves the outcome of prenatally diagnosed complete atrioventricular block without structural heart disease". Circulation 111:e287-e288. doi:10.1161/01.CIR.0000164275.69617.B2, author reply e287-8

44. Sadovsky G, Nicolaides KH (1989) Reference ranges for fetal heart rate patterns in normoxaemic nonanaemic fetuses. Fetal Ther 4:61-68

45. Sanatani S, Potts JE, Reed JH et al (2012) The study of antiarrhythmic medications in infancy (SAMIS): a multicenter, randomized controlled trial comparing the efficacy and safety of digoxin versus propranolol for prophylaxis of supraventricular tachycardia in infants. Circ: Arrhythmia Electrophysiol 5:984-991. doi:10.1161/ CIRCEP.112.972620

46. Schwartz PJ, Salice P (1984) Cardiac arrhythmias in infancy: prevalence, significance and need for treatment. Eur Heart J 5 Suppl B:43-50

47. Southall DP, Johnson AM, Shinebourne EA et al (1981) Frequency and outcome of disorders of cardiac rhythm and conduction in a population of newborn infants. PEDIATRICS 68:58-66 
48. Strasburger JF, Cheulkar B, Wakai RT (2008) Magnetocardiography for fetal arrhythmias. Heart Rhythm 5:1073-1076. doi:10.1016/j. hrthm.2008.02.035

49. Tan HL, Lie KI (2001) Treatment of tachyarrhythmias during pregnancy and lactation. Eur Heart J 22:458-464. doi:10.1053/euhj.2000. 2130

50. Vergani P, Mariani E, Ciriello E et al (2005) Fetal arrhythmias: natural history and management. Ultrasound Med Biol 31:1-6. doi:10.1016/ j.ultrasmedbio.2004.10.001
51. Villain E, Coastedoat-Chalumeau N, Marijon E et al (2006) Presentation and prognosis of complete atrioventricular block in childhood, according to maternal antibody status. J Am Coll Cardiol 48:1682-1687. doi:10.1016/j.jacc.2006.07.034

52. Walsh EP, Triedman JK, Berul CI (1992) Cardiac arrhythmias. In: Keane JF, Lock JE, Fyler DC (eds) Nadas' pediatric cardiology, 2nd edn. Saunders Elsevier, Philadelphia, pp 377-433

53. Yabek SM (1991) Ventricular arrhythmias in children with an apparently normal heart. J Pediatr 119:1-11 